\title{
The Influence of Stray Capacitor on the Harmonic Transmission Characteristics of CVT
}

\author{
Weilun Xie, Feng Xue, Peicheng Xie, Jieting Wu and Dan Peng* \\ Dongguan Power Supply Bureau of Guangdong Power Grid Corporation, Guangdong 523008, China \\ ${ }^{*}$ Corresponding author
}

\begin{abstract}
In view of the fact that the harmonic transmission characteristics of capacitive voltage transformers (CVT) are unclear and not suitable for harmonic voltage measurement, considering the influence of the internal stray capacitance factors, an equivalent circuit model is established and analyzed by transfer function. Stray capacitance has a significant effect on the harmonic transmission characteristics of CVTs. The reference of the distributed capacitance in the CVT is calculated based on the structure of the CVT circuit element in combination with the distributed capacitance derivation. Based on the MATLAB/Simulink simulation tool, the influence of each stray capacitance on the transmission characteristics of various harmonics of CVT was studied, and the relative error caused by stray capacitance change was quantified. Studies have shown that the stray capacitance of the compensation affects the frequency range after the extremum point of the CVT amplitude transfer characteristic, the stray capacitance of the primary side of the intermediate transformer and the coupling capacitance between the primary side and the secondary side affect the entire frequency of the CVT amplitude transfer characteristic. In the segment, the stray capacitance to the ground on the secondary side of the intermediate transformer has no effect on the harmonic transmission characteristics of the CVT.
\end{abstract}

Keywords—capacitor voltage transformer; harmonic; transfer function; frequency characteristics; stray capacitance

\section{INTRODUCTION}

When the CVT is operating at harmonic frequencies, its internal stray capacitance interacts with capacitance and inductance in the main circuit parameters, and unexpected changes to the original model structure of the CVT occur. This will cause the secondary signal of the measurement to have greater error, cannot reflect the actual harmonic level in the electrical network correctly. At present, the research on stray capacitance in CVT focuses on qualitative analysis ${ }^{[1]}$. Reference[2] pointed out that stray capacitance is an indispensable factor in the study of the frequency characteristics of CVT. Reference [3] analyzed the influence of stray capacitance on the CVT amplitude-frequency curve, but it did not deeply analyze the stray capacitance and the error caused by it.

This article analyzes the internal stray capacitance of the CVT and compares the effects of stray capacitance on the harmonic transmission characteristics of the CVT using the transfer function. The reference of the distributed capacitance in the CVT is calculated based on the structure of the CVT circuit element in combination with the distributed capacitance derivation. Based on the MATLAB/Simulink simulation tool, the influence of each stray capacitance on the transmission characteristics of various harmonics of CVT was studied, and the relative error caused by stray capacitance change was quantified.

\section{Equivalent Circuit Model OF CVT}

According to the actual structure of the CVT can be set up its equivalent circuit model shown in Figure 1 in the solid line, Correspondingly, $\mathrm{C}_{1}$ and $\mathrm{C}_{2}$ are the capacitive voltage dividers; $L_{S}$ and $R_{S}$ are the reactance and resistance of the compensation reactor respectively; $\mathrm{L}_{\mathrm{t} 1}$ and $\mathrm{R}_{\mathrm{t} 1}$ are the equivalent leakage impedance of the intermediate transformer primary side; $\mathrm{L}_{\mathrm{t} 2}$ and $\mathrm{R}_{\mathrm{t} 2}$ are the equivalent leakage impedance of the intermediate transformer secondary winding for protection use; $\mathrm{R}_{\mathrm{m}}$ and $\mathrm{L}_{\mathrm{m}}$ are the intermediate transformer excitation impedance; $\mathrm{R}_{b}$ and $\mathrm{L}_{b}$ are the load impedance. When considering the influence of stray capacitance at high frequencies, the equivalent circuit model is shown by the solid line plus dashed line in Figure 1. Correspondingly, $\mathrm{C}_{\mathrm{S}}$ is the stray capacitance of compensation reactor; $\mathrm{C}_{\mathrm{p} 1}$ is the primary side stray capacitance of the intermediate transformer; $\mathrm{C}_{\mathrm{p} 2}$ is the stray capacitance to the ground on the secondary side of the intermediate transformer; $\mathrm{C}_{\mathrm{p} 12}$ is the coupling capacitance between the primary side and the secondary side. CVT is equipped with a damping device, and most of them use a fast saturation damper. When the system is subjected to an overvoltage generated by impulse voltage, a large amount of resonance energy can be consumed in a short time (such as $0.5 \mathrm{~s}$ ) to suppress its own ferromagnetic resonance. Avoid causing the CVT to malfunction or affect the secondary device. The fast saturated damper does not participate in the normal operation of the CVT, so the equivalent impedance of the damper is neglected in the high-frequency equivalent circuit model.

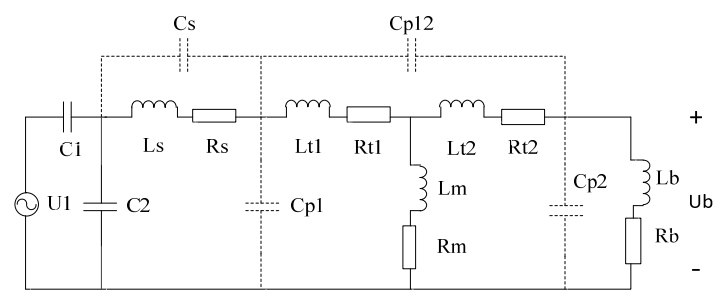

FIGURE I. EQUIVALENT CIRCUIT MODEL OF CVT

In order to further study the effect of stray capacitance on the CVT model, this section will derive the relationship 
between the output voltage of the stray capacitance CVT and the input voltage from the perspective of the transfer function, and plot the transfer function curve of the circuit. CVT intermediate transformer core can be regarded as working in the linear section of the magnetization curve, ignoring the core of the magnetic induction. In the simulation study process, a CVT with a model of TYD $110 / \sqrt{3}-0.01 \mathrm{H}$ was selected as the study object. The main parameters are shown in table 1 .

TABLE I. PARAMETERS OF CVT

\begin{tabular}{|c|c|c|c|}
\hline $\begin{array}{c}\text { Param } \\
\text { eter }\end{array}$ & Value & Parameter & Value \\
\hline $\mathrm{C}_{1}$ & $0.01257 \mu \mathrm{H}$ & $\mathrm{U}_{2}$ & $100 / \sqrt{3} \mathrm{~V}$ \\
\hline $\mathrm{C}_{2}$ & $0.04885 \mu \mathrm{H}$ & $\mathrm{Ls}$ & $164.96 \mathrm{H}$ \\
\hline $\mathrm{U}_{1}$ & $110 / \sqrt{3} \mathrm{kV}$ & $\begin{array}{c}\text { Load capacity } \\
(100 \%)\end{array}$ & $\begin{array}{c}100 \mathrm{VA} / 0 \\
.8\end{array}$ \\
\hline
\end{tabular}

The transfer function of the CVT equivalent circuit model without considering the stray capacitance can be derived from Figure 1, as shown in formula (1).

$$
H_{1}(s)=\frac{Z_{1}}{Z_{1}+Z_{2}+Z_{3}+Z_{4}+Z_{5}}
$$

The transfer function of the CVT equivalent circuit model considering the stray capacitance is derived, as shown in formula (2).

$$
H_{2}(s)=\frac{\left[Z_{1} / / Z_{9}+\left(Z_{2}+Z_{3}\right) / / Z_{8}\right] / / Z_{7}+Z_{4} / / Z_{6}}{\left[Z_{1} / / Z_{9}+\left(Z_{2}+Z_{3}\right) / / / Z_{8}\right] / / Z_{7}+Z_{4} / / Z_{6}+Z_{5}}
$$

Where:

$$
\begin{aligned}
& Z_{1}=s^{*} L_{b}+R_{b} \quad, \quad Z_{2}=s * L_{t 2}+R_{t 2} \quad, \quad Z_{3}=s * L_{t 1}+R_{t 1} \\
& Z_{4}=s * L_{S}+R_{S} \quad, \quad Z_{5}=1 / s\left(C_{1}+C_{2}\right) \quad, \quad Z_{6}=1 / s * C_{S}, \\
& Z_{7}=1 / s * C_{p 1}, \quad Z_{8}=1 / s * C_{p 12}, \quad Z_{9}=1 / s * C_{p 2}
\end{aligned}
$$

Equations (1) and (2) can be obtained through the computer programming transfer function $\mathrm{H}_{1}(\mathrm{~s})$ and $\mathrm{H}_{2}(\mathrm{~s})$ amplitudefrequency characteristics, as shown in Figure 2. It can be seen from the Figure that the CVT amplitude-frequency characteristic curve is monotonically decreasing and the degree of distortion is small when the circuit does not consider the stray capacitance; when considering the stray capacitance, the CVT harmonic measurement curve has severe distortion at the 16th harmonic position. Extremum points appear, that is, CVTs have the effect of amplifying or reducing harmonics of different orders. It can be seen that the stray capacitance has a great influence on the measurement characteristics of the harmonics of the CVT, and the influence of the stray capacitance should be fully taken into account in the measurement and correction of the CVT harmonics.

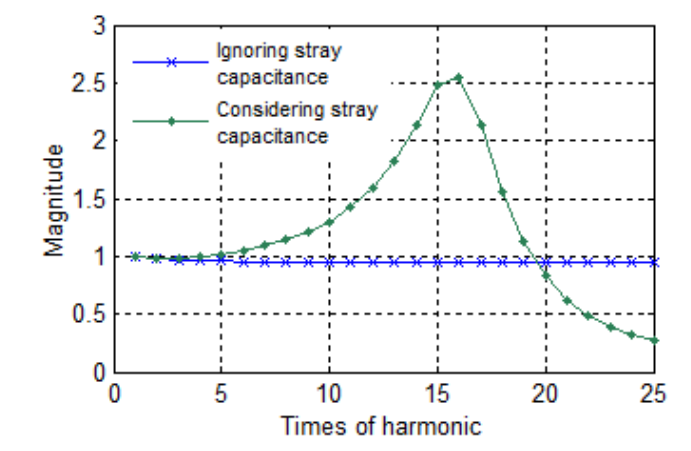

FIGURE II. CVT AMPLITUDE-FREQUENCY CHARACTERISTIC
CURVE

\section{ANALYSIS OF STRAy CAPACITANCE}

According to the capacitance formula of two parallel plates $C=\varepsilon S / d$, after the CVT leaves the factory, the shape and size of the internal components and the relative position between the conductors have been determined, and the ambient temperature and humidity have little influence on them and can be basically ignored. Therefore, it is important to consider that the medium between conductors is affected by environmental factors and affects the stray capacitance. The main dielectric material of the capacitor is insulating oil, which can ignore the influence of humidity, but the influence of temperature on the dielectric constant cannot be ignored. The capacitance temperature coefficient is generally less than or equal to $-4 \times 10^{-}$ $4 / \mathrm{K}$. When the ambient temperature changes $\pm 40^{\circ} \mathrm{C}$, it may bring about a change of $3.2 \%$ in dielectric constant, which will directly affect the accuracy of harmonic transmission of CVT. At the same time, for different types of CVTs, stray capacitance values are different and are not stable values, but stray capacitances can be estimated through known structural parameters. This will make sense for follow-up studies of CVTs.

\section{A. Compensation Reactor Stray Capacitance}

From the previous analysis, it can be seen that the change in the dielectric constant causes about 3\% error. The compensating reactor has an adjustable air gap, and each tap has an adjustable amount of $\pm 2 \%$, causing the variation of the equivalent stray capacitance $\mathrm{C}_{S}$ of the compensating reactor to be about $\pm 2 \%$. Consider the change in $\mathrm{C}_{\mathrm{S}}$ overall within $\pm 5 \%$.

In [4], the electromagnetic field theory is used to analyze the distributed capacitance of the U-shaped row winding induction coil. The results show that for the reactor of the nlayer winding, the equivalent distributed capacitance is shown in Equation (3):

$$
C_{x}=\frac{4(n-1) \varepsilon_{r} \varepsilon_{0} l D N}{3 n^{2} d}
$$

Where: 1 is the average length, $\mathrm{D}$ is the diameter of the conductor cross-section, $\mathrm{d}$ is the distance between layers, and $\mathrm{N}$ is the number of turns in each layer. The stray capacitance of 
the compensating reactor is calculated as $80.3 \mathrm{pF}$, as shown by the commonly used compensation reactor parameters provided by a factory $(n=10$ layers, $N=300,1=82.4 \mathrm{~mm}, D=0.25 \mathrm{~mm}$, $\mathrm{d}=0.18 \mathrm{~mm}, \varepsilon_{r}=2.2$ ) and in combination with formula (3).

\section{B. Distributed Capacitance Parameters of Intermediate Transformer Windings}

The middle transformer of CVT will change the intermediate voltage of $13 \sim 20 \mathrm{kV}$ into the secondary voltage. The core uses high quality cold-rolled silicon steel sheet to improve the antiferromagnetic resonance characteristics. For $\mathrm{C}_{\mathrm{p} 1}, \mathrm{C}_{\mathrm{p} 2}$, and $\mathrm{C}_{\mathrm{p} 12}, \pm 5 \%$ of the regulation winding is designed for the primary winding of the intermediate transformer. Consider $\mathrm{C}_{\mathrm{p} 1}, \mathrm{C}_{\mathrm{p} 2}$, and $\mathrm{C}_{\mathrm{p} 12}$ variations over a wide range. Take $\pm 10 \%$ of the study here.

According to the capacitance divider composed of $\mathrm{C}_{1}$ and $\mathrm{C}_{2}$, the primary winding voltage of the intermediate transformer can be calculated as $\mathrm{Up}=\mathrm{U}_{1} \mathrm{C}_{1} /\left(\mathrm{C}_{1}+\mathrm{C}_{2}\right)=12997.4 \mathrm{~V}$, and the rated secondary voltage of the CVT is $100 / \sqrt{3} \mathrm{~V}$. At present, the potential of number of turns $(\mathrm{T})$ of the intermediate transformer is generally taken $\mathrm{e}_{\mathrm{t}}=1.3 \mathrm{~V} / \mathrm{T}$. From this, it can be seen that the primary winding of intermediate transformer $\mathrm{N}_{1} \approx 10000 \mathrm{~T}$, the secondary winding of intermediate transformer $\mathrm{N}_{2} \approx 45 \mathrm{~T}$.

The intermediate transformer winding and the compensation have a similar structure, so the distributed capacitance parameters of the intermediate transformer winding can also be calculated with reference to formula (3), including the primary side-to-ground stray capacitance of the intermediate transformer and the secondary side-to-ground stray capacitance. The primary winding parameters are: $\mathrm{n}=16$ layers, $\mathrm{N}=650, \mathrm{l}=58.6 \mathrm{~mm}, \mathrm{D}=0.25 \mathrm{~mm}, \mathrm{~d}=0.18 \mathrm{~mm}, \quad \varepsilon_{r}=2.2$; the primary winding parameters are: $\mathrm{n}=2$ layers, $\mathrm{N}=25$, $\mathrm{l}=49.4 \mathrm{~mm}, \mathrm{D}=0.25 \mathrm{~mm}, \mathrm{~d}=0.18 \mathrm{~mm}, \varepsilon_{r}=2.2$. It can be seen that $\mathrm{C}_{\mathrm{p} 1}=80.5 \mathrm{pF}, \mathrm{C}_{\mathrm{p} 2}=11.1 \mathrm{pF} ; \mathrm{C}_{\mathrm{p} 12}$ is assumed to be $80 \mathrm{pF}$.

\section{CAPACITANCE EFFECTS ON CVT}

This section builds a MATLAB/Simulink simulation model based on the CVT equivalent circuit model, as shown in Figure 3. The stray capacitance calculated above is taken as a reference value into the model, and its internal stray capacitance parameters are simulated and analyzed.

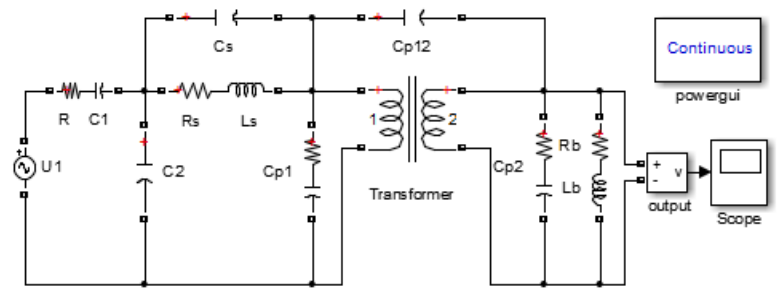

FIGURE III. SIMULINK MODEL OF CVT

\section{A. Effect of Compensating Reactor Equivalent Stray Capacitance}

Figure 4 shows the CVT ratio transfer characteristic curve and error curve when Cs changes by $\pm 5 \%$. From an intuitive point of view, changes in $\mathrm{C}_{\mathrm{S}}$ can affect the amplitude transfer characteristics of CVT, and have little effect on the harmonic transformation ratio before the extreme point, resulting in a maximum variation error of only $1 \%$. The harmonic transformation ratio after the extreme point has a great influence, and can reach $8 \%$ at the 23 rd harmonic.

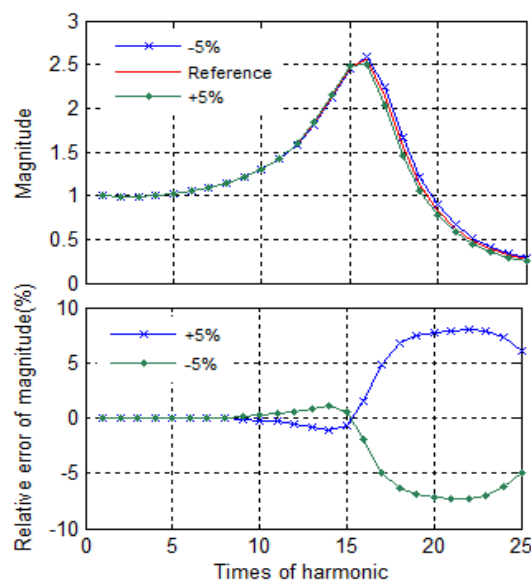

FIGURE IV. CVT AMPLITUDE-FREQUENCY CHARACTERISTIC AND ERROR WHEN $\mathrm{C}_{\mathrm{S}}$ CHANGES WITHIN $\pm 5 \%$

\section{B. Distributed Capacitance Parameters of Intermediate Transformer Windings}

When $\mathrm{C}_{\mathrm{p} 1}$ changes by $\pm 10 \%$, the CVT transfer curve is plotted, as shown in Figure 5. Intuitively, changes in $\mathrm{C}_{\mathrm{S}}$ affect the amplitude transfer characteristics of CVTs, affecting the harmonic transformation ratio prior to the extreme point of around $5 \%$. The harmonic transformation ratio after the extremum point has a great influence, reaching $7.8 \%$ at the 19th harmonic. 


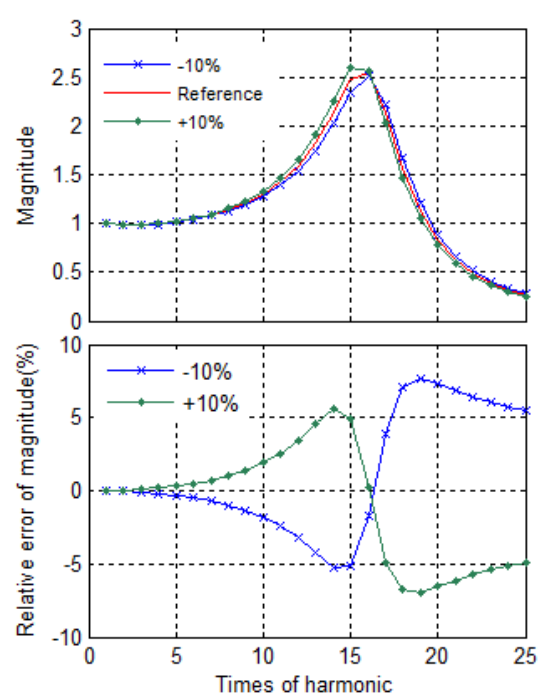

FIGURE V. CVT AMPLITUDE-FREQUENCY CHARACTERISTIC AND ERROR WHEN $\mathrm{C}_{\mathrm{pl}}$ CHANGES WITHIN $\pm 10 \%$

When $\mathrm{C}_{\mathrm{p} 12}$ changes at $\pm 10 \%$, the CVT transmission curve is plotted, as shown in Figure 6. It can be seen that the change of $\mathrm{C}_{\mathrm{p} 12}$ will affect the amplitude transfer characteristics of CVT, and the influence of $\mathrm{C}_{\mathrm{p} 1}$ on the amplitude transfer characteristics is similar, and the harmonic transformation ratio before the extreme point affects about 5\%. The harmonic transformation ratio after the extremum point has a great influence, reaching $7.8 \%$ at the 19 th harmonic.

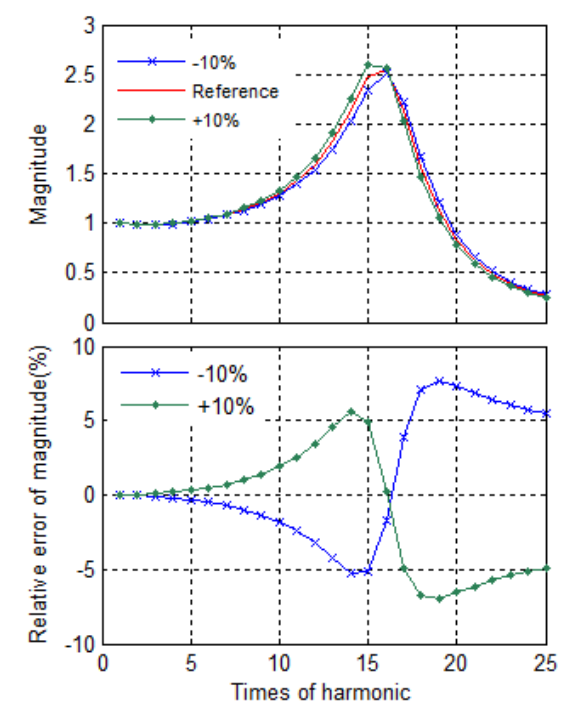

FIGURE VI. CVT AMPLITUDE-FREQUENCY CHARACTERISTIC AND ERROR WHEN $\mathrm{C}_{\mathrm{p} 12}$ CHANGES WITHIN $\pm 10 \%$

When $\mathrm{C}_{\mathrm{p} 2}$ is changed by $\pm 10 \%$, the CVT transfer characteristic curve when $C_{p 1}$ changes is plotted, as shown in Figure 7. It can be seen that the $\mathrm{C}_{\mathrm{p} 2}$ change has no effect on the amplitude transfer characteristics of the CVT.

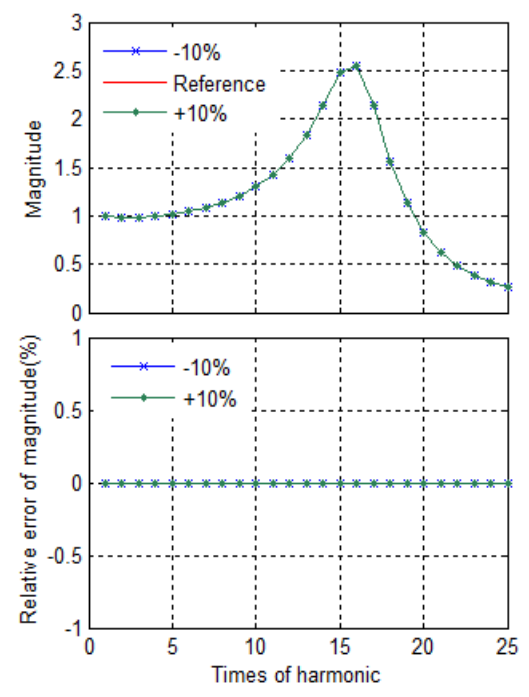

FIGURE VII. CVT AMPLITUDE-FREQUENCY CHARACTERISTIC AND ERROR WHEN $\mathrm{C}_{\mathrm{p} 2}$ CHANGES WITHIN $\pm 10 \%$

\section{CONCLUSION}

This article deeply analyzes the stray capacitance characteristics and deduces its reference value theoretically. It calculates the harmonic transmission characteristics of CVT and completes the simulation verification work. The main conclusions are as follows:

Considering the influence of stray capacitance, the extreme value point of the amplitude and frequency characteristic curve of CVT will appear, leading to a larger measurement error;

$\mathrm{C}_{\mathrm{S}}$ affects the frequency range after the extreme value point of the CVT amplitude transfer characteristic. $\mathrm{C}_{\mathrm{p} 1}$ and $\mathrm{C}_{\mathrm{p} 12}$ affect the entire frequency range of the CVT amplitude transfer characteristics, while $\mathrm{C}_{\mathrm{p} 2}$ does not have any effect on the harmonic transmission characteristics of the CVT.

The maximum influence error of $\mathrm{C}_{\mathrm{S}}$ at $\pm 5 \%$ is $8 \%$, and the maximum influence error of $\mathrm{C}_{\mathrm{p} 1}$ and $\mathrm{C}_{\mathrm{p} 12}$ within $\pm 10 \%$ is $7.8 \%$, which shows that $\mathrm{C}_{\mathrm{S}}$ has more significant influence on the harmonic transmission characteristics of CVT.

Therefore, when studying the harmonic transmission characteristics of CVT, the effects of stray capacitance, primary stray capacitance, and secondary-side coupling capacitance of the compensation reactor should be fully considered.

\section{ACKNOWLEDGMENT}

This work was supported by the Special Funds for Dongguan Power Supply Bureau of Guangdong Power Grid Corporation (No.607170339). Thus, the authors gratefully acknowledge Southern Power Grid of China for providing assistance and technical support.

\section{REFERENCES}

[1] Foroozan Ghassemi, Philip Gale, Tom Cumming, Colin Coutts. "Harmonic Voltage Measurements Using CVTs," J. IEEE transactions on Power Delivery, 2005, vol. 1:443-449. 
[2] Hongliang Gao, Qionglin Li, Xiaopeng Yu, Zhenan Zhang, Shuangyin Dai, Shuming Liu. "The study of Capacitive voltage transformer harmonic transmission characteristics,". Power System Technology, vol. 37, pp. 3125-3130, November 2013.

[3] Li Zhao; Jianchun Wei; Qingquan Li; Guangke Xu; Yufeng Chen; Bin Liu; Baohua Wang; Zhenxin Qi. "The Influence of CVT Parameters on the Harmonic Transmission Characteristics," IEEE 11th Conference on Industrial Electronics and Applications (ICIEA), 2016

[4] Chen Long, Zhao Yanmei, Zhang Ning. "A Simplified Model to Calculate the Distributed Capacitance of Inductor Coil," J. Journal of Nanjing Normal University (Natural Science Edition), vol. 37, pp. 55-59, 2014. 